\title{
Distribución y dinámica poblacional de la almeja asiática Corbicula fluminea (Bivalvia, Corbiculidae) en ríos de Uruguay
}

\author{
Marcel Rodríguez ${ }^{1, \otimes} ;$ Pablo Muniz ${ }^{1,2} ;$ Alejandro Brazeiro $^{3}$ \& Omar DefeO ${ }^{2 / 4}$ \\ ${ }^{1}$ Sección Oceanografía y Ecología Marina, Instituto de Ecología y Ciencias Ambientales, Facultad de Ciencias, Universidad \\ de la República (UdelaR). ${ }^{2}$ Grupo de Estudios Pesqueros y de Impacto Ambiental (GEPEIA), CURE-UdelaR. ${ }^{3}$ Grupo \\ Biodiversidad y Ecología de la Conservación (BEC), Instituto de Ecología y Ciencias Ambientales, Facultad de Ciencias, \\ Universidad de la República (UdelaR). ${ }^{4}$ Unidad de Ciencias del Mar (UNDECIMAR), Facultad de Ciencias, Universidad de la \\ República (UdelaR).
}

\begin{abstract}
Resumen. La almeja asiática (Corbicula fluminea) es un bivalvo originario del sudeste asiático, introducido en la cuenca del Río de la Plata a fines de la década del sesenta. Desde entonces invadió numerosos cursos de agua en Brasil y la Argentina, hasta llegar a Venezuela y Colombia; actualmente, su distribución en Uruguay es poco conocida. En este trabajo analizamos la distribución espacial y características poblacionales de C. fluminea en las cuencas de las regiones Centro-Sur y Este de Uruguay, en relación con variables abióticas. Se relevó la presencia-ausencia de C. fluminea bimestralmente durante un año y se estimó la abundancia, el crecimiento y la mortalidad en cinco sitios en la cuenca del río Santa Lucía. La incidencia de la almeja asiática en la región fue de $50 \%$. La probabilidad de ocurrencia y abundancia aumentaron con el porcentaje de arena en el sedimento y disminuyeron con el porcentaje de grava. En sedimentos finos (limos y arenas) se observó un crecimiento lento, grandes tamaños corporales y baja mortalidad, mientras que en sedimentos gruesos (gravas), los patrones siguieron una tendencia opuesta. Los parámetros de crecimiento y la mortalidad no se correlacionaron con la densidad, lo que sugiere que esta población estaría regulada primariamente por factores ambientales. $C$. fluminea podría continuar su expansión en Uruguay invadiendo sectores arenosos de los cursos de agua con bajos porcentajes de grava y limos.
\end{abstract}

[Palabras clave: invasión, regulación poblacional, relación bentos-sedimento]

\begin{abstract}
Distribution and population dynamics of the Asiatic clam Corbicula fluminea (Bivalvia, Corbiculidae) in rivers of Uruguay. The Asian clam (Corbicula fluminea) is a bivalve native to southeast Asia, introduced into the River Plate Basin to the end of the sixties. Since then, it has invaded a large number of watercourses in Brazil and Argentina, until reaching Venezuela and Colombia; its current distribution in Uruguay is poorly known. In this paper, we analyze the spatial distribution and population characteristics of C. fluminea in the basins of the South-Central and Eastern regions of Uruguay, in relation to abiotic variables. Sampling was conducted during one year in a bimonthly basis, and abundance, growth and mortality were estimated in five sites in the Santa Lucía river basin. The incidence of the Asian clam in the region was 50\%. The probability of occurrence and abundance increased with the percentage of sand in the sediment and decreased with that of gravel. Slow growth rates, high body sizes and low mortalities were observed in fine sediments (sands and silts), following opposite trends in coarse sediment (gravels). Growth and mortality parameters were not correlated with density, suggesting that this population would be primarily regulated by environmental factors. C. fluminea could continue its expansion in Uruguay, invading sandy sectors of watercourses with low percentages of gravel and silt.
\end{abstract}

[Keywords: invasion, population regulation, benthos-sediment relationship]

\section{INTRODUCCIÓN}

Todos los organismos se dispersan en algún grado en la naturaleza. Se puede definir la dispersión como el proceso natural mediante el cual las especies pueden colonizar nuevas áreas y los individuos escapan del ambiente inmediato de sus vecinos (Begon et al. 1996). La dispersión puede ser un proceso activo, como en el caso de ciertas especies de aves y roedores que tienen medios de locomoción propios (Shigesada and Kawasaki 1997), o puede ocurrir en forma pasiva, mediada

Editora asociada: Claudia Feijoo

\marcel@fcien.edu.uy por agentes físicos o biológicos (Begon et al. 1996).

El proceso de globalización ha facilitado el transporte de organismos a través de las barreras geográficas que habían mantenido separadas las regiones del planeta, por lo que muchas de las especies de actual importancia ecológica fueron introducidas en tiempos históricos (Chapin et al. 2000; Strauss et al. 2006). Las actividades humanas también volvieron obsoletas las barreras para la dispersión de plantas terrestres al

Recibido: 24 de Septiembre de 2020

Aceptado: 3 de Marzo de 2021 
incrementar la presión de propágulos de plantas no nativas a nivel mundial (Mazia et al. 2001). En el ambiente marino, la frecuencia de transporte de organismos aumentó en las últimas décadas debido al aumento del tráfico y a la globalización del comercio (Morton 1977; Palacios et al. 2000; Crooks 2002; Hoffmann and Courchamp 2016).

Una invasión biológica ocurre cuando una especie coloniza un área en la cual no habitaba anteriormente y persiste en ella causando una variedad de impactos en las condiciones ambientales, sanitarias y económicas de las regiones invadidas (Shigesada and Kawasaki 1997; Simberloff et al. 2012). De este modo, la invasión de hábitats por animales y plantas no nativos se convirtió en un fenómeno de alcance global, con consecuencias potencialmente graves a nivel de comunidades y ecosistemas, pero también a nivel social y económico (Sakai et al. 2001; Strauss et al. 2006). Si bien numerosas especies invasoras, como por ejemplo el mejillón cebra (Dreissena polymorfa) (Morton 1973) en América del norte y la rata almizclera (Ondartra zibethica) en Europa (Shigesada and Kawasaki 1997), han recibido mayor atención, tanto en la prensa popular como en la comunidad científica (Davis and Thompson 2000), el estudio de la invasión biológica y de las especies invasoras no requiere herramientas diferentes que el estudio de las especies pioneras y la colonización de un nuevo hábitat. El arribo de una especie no nativa a un nuevo ambiente y la llegada de una especie pionera a un hábitat recientemente perturbado son básicamente el mismo fenómeno, pues en ambos casos se está adaptando a un nuevo sitio (Davis et al. 2000; Hoffmann and Courchamp 2016). La invasión de un ambiente por una nueva especie está influenciada por tres factores: el número de propágulos que llegan al nuevo ambiente, las características de la nueva especie (invasiveness) y la susceptibilidad del ambiente a la invasión por nuevas especies. Este último factor es conocido como invasibilidad (invasibility) y es una propiedad emergente de un ambiente, resultado de la interacción de muchos factores, incluyendo, entre otros, el clima de la región, el régimen de disturbios y las habilidades competitivas de las especies presentes (Davis et al. 2000; Darrigran and Damborenea 2011; Bresciano et al. 2014). Las características del nuevo invasor/colonizador dependen de su filogenia. La familia Corbiculidae (=Cyrenidae) comprende los géneros Batissa, Polymesoda, Cyanocyclas y Corbicula. Los dos primeros géneros son principalmente de agua salina, característicos de los sedimentos reducidos de los manglares. Solo Cyanocyclas y Corbicula poseen una cantidad importante de especies de agua dulce (Parodiz and Hennings 1963; Britton and Morton 1982). El género Corbicula Megerle 1811, comprende numerosas especies, originarias del Sudeste de Asia. La especie C. fluminea es invasora en América y es conocida popularmente como 'almeja asiática', una invasora (sensu Davis and Thompson 2000) capaz de causar efectos en la trama trófica, el ecosistema y a nivel social y económico (Morton 1977; VentenheimerMendes and Olazarri 1983; Amestoy et al. 1998; Sousa et al. 2009; Foster et al. 2012; Morales et al. 2013).

La almeja asiática fue introducida en el Río de la Plata a fines de la década del sesenta. En un primer momento, sobre la base a caracteres conquiliológicos y de anatomía blanda, se reconoció la presencia de dos especies: Corbicula leana Prime 1864 y Corbicula fluminea Müller 1774 (Ituarte 1981). Estudios posteriores confirmaron la presencia de $C$. fluminea y demostraron que la segunda especie no era C. leana sino Corbicula largillierti Philippi 1844 (Ituarte 1982). La primera cita para esas aguas corresponde al año 1979 (Ituarte 1981) en Punta Lara, aunque es imposible precisar la fecha exacta de su introducción debido a los escasos estudios orientados al conocimiento de las comunidades bentónicas del Río de la Plata en la época. Los últimos muestreos en los que no se encontró $C$. fluminea ni $C$. largillierti fueron llevados a cabo por Parodiz en 1961 para los ríos Uruguay y Paraná, y por Castellanos en 1965 para el Río de la Plata (Ituarte 1981), por lo cual el arribo de ambas especies a este último se sitúa en algún punto entre mediados de los sesenta y fines de los setenta. En Uruguay, la última colecta en la cual estas especies están ausentes corresponde al año 1968 en la bahía de Colonia (Ventenheimer-Méndez and Olazarri 1983).

Durante el año 1979, Ituarte (1981) señala que el género Corbicula ocupa una franja continua en el litoral del Río de la Plata argentino desde su naciente hasta el Balneario Magdalena y Atalaya, y no se encontraba en arroyos afluentes al mismo. Esta distribución correspondía a C. largillierti; C. fluminea se encontraba solamente al norte del puerto de Buenos Aires. Durante el 1982, C. fluminea se la encuentra en Atalaya, en simpatría con $C$. largillierti. En el año 1985 Darrigran (1992a) cita la presencia de $C$. fluminea en arroyos afluentes 
al Río de la Plata. Darrigran (1992b) cita la presencia de C. fluminea en la franja descripta por Ituarte, en simpatría con C. largillierti pero con tres diferencias: 1) en algunas localidades no existía la presencia de C. largillierti; 2) en la que había simpatría de ambas especies, la densidad de C. fluminea era mayor a la de C. largillierti. 3) C. fluminea se extendió en su dispersión más que $C$. largillierti, hasta llegar a Punta Indio, donde el cambio de salinidad comienza a manifestarse en el litoral rioplatense. En 1983, la distribución de ambas especies en la costa uruguaya del Río de la Plata se presentaba discontinua, con citas aisladas para los departamentos de Colonia y San José (Veitenheimer-Mendes and Olazarri 1983). Para 1986, la presencia de las dos especies estaba confirmada en el río Uruguay desde su desembocadura hasta la ciudad de Paysandú, en el río San Salvador desde su boca hasta sus nacientes y en el río Negro desde su desembocadura en el río Uruguay hasta la represa de Palmar (Olazarri 1986). En 1994, C. fluminea y C. largillierti ya habían llegado por el Paraná más allá de su encuentro con el río Paraguay (Ituarte 1994). En 1998, las dos especies podían encontrarse en el río Uruguay hasta su confluencia con el río Cuareim, y en el Río de la Plata desde el delta del río Paraná hasta la desembocadura del río Santa Lucía (Amestoy et al. 1998). En Brasil, C. fluminea fue encontrada en los ríos Jacuí y Guaiba, en la zona norte de la Laguna de los Patos (Veitenheimer-Mendez 1981) y posteriormente en el bañado de Taim, en la cuenca de la laguna Merín (Mansur and Garces 1988). Registros más recientes indican que Corbicula avanzó hasta el río Chapecó, un afluente del río Uruguay superior, en el estado de Santa Catarina (Agudo-Padrón 2017). Asimismo, C. fluminea se extendió ampliamente e invadió las principales cuencas hidrológicas de la Región Neotropical. En 1988 fue registrada por primera vez en la cuenca amazónica brasileña (01⒌' S - 543' O), y actualmente se distribuye desde el Río Colorado $\left(39^{\circ} 01^{\prime} S\right.$ - 6401' O), en el límite norte de la Patagonia

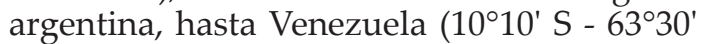
O) (Cao et al. 2018).

Actualmente, no es mucho más lo que se conoce sobre la distribución de C. fluminea en Uruguay. Según Rodríguez y Palacios (2001), C. fluminea ingresó en el río Santa Lucía, superó las represas del río Negro y se encuentra en la costa uruguaya de la laguna Merín. De este modo se hace clara la necesidad de determinar de forma precisa cuál es el alcance de la invasión de C. fluminea en Uruguay y su mecanismo de dispersión. En Uruguay aún no se han presentado los problemas de obstrucción de canales de riego (Cherry et al. 1986; Morton 1977; Cherry et al. 1980; Isom 1986), tomas de agua y plantas hidroeléctricas (Cherry et al. 1980; Isom 1986), aunque todavía no está claro si esto se debe a alguna limitante ambiental o biológica, o simplemente al hecho de que la introducción de C. fluminea en el Río de la Plata es aún demasiado reciente (Ituarte 1981; Darrigran and Maroñas 1989; Darrigran 1992b). Sólo hubo unos pocos informes del impacto económico de la especie en Sudamérica durante 1998, en una central hidroeléctrica ubicada en Rio Grande do Sul (Reshaid et al. 2017). Otro molusco introducido, el mejillón asiático dorado Limnoperna fortunei, ya ha causado problemas en la planta potabilizadora de Aguas Corrientes (Clemente and Brugnoli 2002; Brugnoli et al. 2005; Langone 2005a; Muniz et al. 2005). Según Britton y Morton (1982), C. fluminea presenta amplios rangos de tolerancia en cuanto a sustratos y corrientes, por lo que no habría límites para su expansión en los cursos de agua de Uruguay. Esto es sumamente preocupante, ya que algunos de los cursos y cuerpos de agua amenazados son de gran importancia socioeconómica, dado que se usan como fuente de agua para riego y consumo humano.

La tasa de crecimiento y la producción de gametos son buenos indicadores de la adecuación del ambiente a los requerimientos de un organismo, ya que representan la respuesta integrada de las actividades fisiológicas del mismo (McDonald and Thompson 1985). De tal forma, la estimación de aspectos demográficos y de la dinámica poblacional de C. fluminea, en conjunto con el estudio de sus relaciones con variables ambientales, indicaría cuán adecuado o no es un sitio para la especie, o si existen limitantes ambientales para su distribución. Por tanto, el objetivo del presente trabajo fue mapear la presencia de C. fluminea en las zonas CentroSur y Este de Uruguay, y evaluar el efecto de la textura del sedimento, la temperatura, la concentración de oxígeno disuelto, la materia orgánica en suspensión (MOS) y la salinidad en su ocurrencia, densidad poblacional, crecimiento y mortalidad. Asimismo, se evaluó la posible existencia de densodependencia en el crecimiento y la mortalidad. 


\section{Materiales y Métodos}

\section{Área de estudio}

El área de estudio comprendió la cuenca de la Laguna Merín, parte de la cuenca del río Negro y la cuenca del río Santa Lucía (Figura 1). La extensa Laguna Merín está ubicada en la frontera entre Brasil y Uruguay (Barone et al. 1994). Sus costas están constituidas por playas de arena de escasa profundidad y extensos humedales. Los principales afluentes son los ríos Cebollatí, Tacuarí y Yaguarón (Daroczi et al. 1983; Barone et al. 1994) (Figura 1). El río Negro nace en el Nudo de Santa Tecla, en Brasil, para luego cruzar el territorio uruguayo en dirección este-oeste (OPP, OEA and BID 1992), desembocando en el río Uruguay (Figura 1). Sus principales afluentes son los ríos Tacuarembó y Yí (Barone et al. 1994). Sus centrales hidroeléctricas, junto con la central binacional de Salto Grande, forman la base del suministro de energía eléctrica en el país. El río Santa Lucía es el principal curso de agua al sur del país. Nace en las sierras del departamento de Lavalleja y desemboca en el Río de la Plata (Figura 1). Sus principales afluentes son los ríos Santa Lucía chico y San José, pero desembocan en él más de 200 arroyos y 1000 cañadas (OPP, OEA and BID 1992). En la localidad de Aguas Corrientes se encuentra la central potabilizadora de agua de Obras Sanitarias del Estado (O.S.E.), que, aprovechando el cauce del río, abastece de agua potable a la ciudad de Montevideo y a gran parte del sur del país (Kurucz et al. 1998).

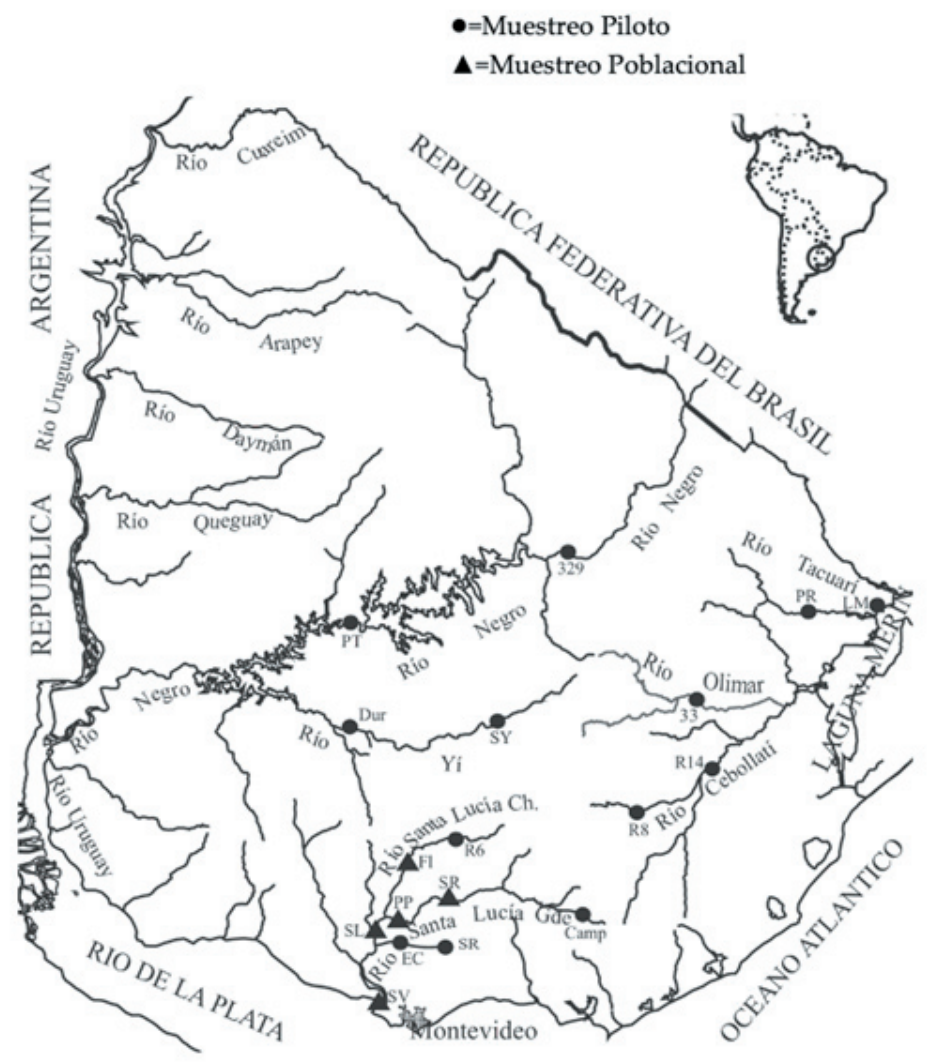

Figura 1. Mapa del área de estudio mostrando la ubicación de los sitios de muestreo. Todos los sitios fueron visitados durante el muestreo piloto. Los sitios en la cuenca del río Santa Lucía fueron revisitados durante los muestreos poblacionales. SV: Santiago Vásquez; PP: Paso Pache; Fl: Florida; PR: Plácido Rosas; PT: Paso de los Toros; LM: Laguna Merín; Sram: San Ramón; SL: Santa Lucía; 329: km 329; Camp: Arroyo Campanero; Ecan: Embalse del Canelón Grande; SR: Santa Rosa; SY: Sarandi del Yí; R6: Santa Lucía Chico sobre la ruta 6; R14: Cebollatí sobre la ruta 14; R8: Cebollatí sobre la ruta 8; Dur: Durazno.

Figure 1. Map of the study area showing the location of the sampling sites. All the sites were visited during the pilot sampling. Sites in the Santa Lucía river basin were revisited during the populational samplings. SV: Santiago Vásquez; PP: Paso Pache; Fl: Florida; PR: Plácido Rosas; PT: Paso de los Toros; LM: Merín Lagoon; Sram: San Ramón; SL: Santa Lucía; 329: km 329; Camp: Arroyo Campanero; Ecan: Embalse del Canelón Grande; SR: Santa Rosa; SY: Sarandi del Yí; R6: Santa Lucía Chico on route 6; R14: Cebollatí on route 14; R8: Cebollatí on route 8; Dur: Durazno 


\section{Muestreo}

Muestreo piloto. Entre febrero y junio de 2001 se realizó un muestreo piloto en diversos cursos de agua de las zonas Centro-Sur y Este de Uruguay (Figura 1) para determinar la distribución de $C$. fluminea. Cada curso de agua se muestreó en al menos dos puntos, uno en su curso bajo y otro en el curso medio o superior, excepto en el río Olimar, el Tacuarí y la Laguna Merín, en los cuales se realizó un único muestreo debido a causas logísticas. En el muestreo piloto, los mayores valores de densidad poblacional se encontraron en la cuenca del río Santa Lucía y en particular en los ríos Santa Lucía grande y Santa Lucía chico, por lo cual los posteriores muestreos poblacionales se centraron en dicha cuenca.

Muestreos poblacionales. Una vez procesada la información del muestreo piloto, el estudio se centró en la cuenca del río Santa Lucía (Figura 1). Los muestreos se realizaron cada dos meses entre junio 2001 y setiembre de 2002, en 5 sitios de la cuenca del río Santa Lucía: Barra del Santa Lucía, Ciudad de Santa Lucía, Paso Pache, Florida y San Ramón (Figura 1). Durante los meses de verano, desde noviembre de 2001 a febrero de 2002, los muestreos se realizaron mensualmente para aumentar la resolución en la época de mayor crecimiento esperado. Los cinco sitios se eligieron por ser fácilmente accesibles, por haber presentado altas densidades de individuos en el muestreo piloto y por presentar subambientes claramente diferenciables (orilla, canal, banco). Los 5 sitios también representaron un gradiente de condiciones ambientales desde la desembocadura del río Santa Lucía hacia sus nacientes.

Las muestras se extrajeron con un corer de $16 \mathrm{~cm}$ de diámetro $\left(200 \mathrm{~cm}^{2}\right)$. El material obtenido fue tamizado (malla de $1 \mathrm{~mm}$ ) en el campo para separar las almejas del sedimento. En cada sitio se consideraron tres subsitios: la orilla, el canal y el banco de arena, tomándose cuatro repeticiones en cada uno para determinar la densidad poblacional. Para estimar el crecimiento individual, en el subsitio con mayor cantidad de almejas de cada sitio se continuó extrayendo ejemplares hasta completar 200 individuos. Las almejas fueron conservadas en hielo hasta su llegada al laboratorio.

En cada sitio se midió la temperatura, la concentración de oxígeno disuelto y la salinidad con un multiparámetro Horiba.
También se tomaron tres muestras de sedimento con un corer de $16 \mathrm{~cm}$ de diámetro para su análisis granulométrico, y una muestra de agua directamente con una botella de 1 litro, para determinar la materia orgánica en suspensión.

\section{Análisis de laboratorio}

En el laboratorio, las almejas fueron congeladas y posteriormente se midió el largo de la valva (L) con un calibre Mitutoyo $( \pm 0.01 \mathrm{~mm})$. Las muestras de sedimento fueron analizadas mediante el método de tamizado descrito por Sugio (1973) a fin de calcular el diámetromedio de grano $(\mathrm{Md})$ y los porcentajes de las distintas fracciones granulométricas (grava, arena, limo y arcilla) (Folk and Ward 1957). Para determinar la concentración de materia orgánica en suspensión, las muestras de agua se sometieron al procedimiento de filtrado, secado y pesado descrito por Arocena y Conde (1999).

\section{Análisis de datos}

Con los datos obtenidos en el muestro piloto, se mapeó la distribución de C. fluminea en las zonas Centro-Sur y Este de Uruguay (Figura 1). También se realizaron regresiones logísticas de presencia-ausencia contra fracciones granulométricas del sedimento $(\%)$, temperatura $\left({ }^{\circ} \mathrm{C}\right)$, concentración de $\mathrm{O}_{2}(\mathrm{mg} / \mathrm{L})$ y materia orgánica en suspensión $(\mathrm{mg} / \mathrm{L})$.

Las variables ambientales medidas en los muestreos poblacionales fueron comparadas entre los cinco sitios utilizando un ANOVA de una vía para medidas repetidas. Cuando se observaron diferencias significativas entre sitios, se las determinó a través de la prueba $a$ posteriori LSD (Zar 1996).

Se calculó la densidad para cada subsitio como el promedio de las densidades de los cuatro corers obtenidos en el mismo. La densidad poblacional en cada sitio de muestreo se calculó como el promedio de las densidades de los doce corers obtenidos en cada sitio. Siguiendo el mismo criterio que para las variables abióticas, para evaluar diferencias en densidad entre sitios se empleó un ANOVA anidado (subsitio anidado en sitio). Los datos de densidad fueron sometidos a una transformación logarítmica [y $\left.{ }^{\prime}=\log (\mathrm{y}+1)\right]$, debido a que la distribución de los datos mostró una asimetría positiva. Esta transformación comprime valores altos y dispersa los valores bajos, acercando la 
distribución de los datos a una distribución normal. La prueba LSD a posteriori fue utilizada cuando se encontraron diferencias significativas (Zar 1999).

La clave largo-edad se obtuvo mediante el método de Bhattacharya (Pauly and Caddy 1985) y la rutina Linking of Means del programa FISAT II (Gayanilo et al. 2002). A partir de esta última, por métodos de optimización no lineales, se ajustó la ecuación generalizada de crecimiento de von Bertalanffy (ECVB) propuesta por Pauly y Gaschütz (1979) y modificada por Somers (1988) para estimar la estacionalidad en el crecimiento. Las ECVB ajustadas para cada sitio se compararon a través de la prueba de cociente de máxima verosimilitud (LRT) (Kimura 1980). Para realizar el LRT, se compararon simultáneamente los cinco parámetros de la ECVB (largo infinito: Lo; constante de crecimiento: $\mathrm{K}$; tiempo subcero: $\mathrm{t}_{0}$; parámetro de estacionalidad: $\mathrm{C}$; winter point: WP) en los cinco sitios, tomando como hipótesis nula general que no diferían significativamente entre sitios. Posteriormente, se fue alterando de forma secuencial el número de parámetros comparados hasta lograr todas las combinaciones de parámetros posibles (Haddon 2001).

La tasa instantánea de mortalidad natural (M) se calculó mediante curvas de captura convertidas a longitudes (Lengthconverted Catch Curves: LCCC) empleando el programa FISAT II (Gayanilo et al. 2002). Esta aproximación permitió la estimación de la mortalidad total $\mathrm{Z}$ (en este caso, $\mathrm{Z}=\mathrm{M}$ ya que $C$. fluminea no está sometida a presión de pesca).

Para evaluar cómo las variables ambientales afectaron la densidad, el crecimiento y la mortalidad se realizaron regresiones lineales múltiples considerando los promedios por muestreo para cada sitio como entradas en el análisis. Los datos fueron transformados para cumplir con los supuestos de linealidad y normalidad, y se realizó un análisis de residuales para determinar la adecuación de las transformaciones. Para evaluar el posible efecto de la densidad sobre el crecimiento y la mortalidad se realizaron regresiones lineales de los parámetros de la ECVB, como variables dependientes, sobre la densidad promediada para cada sitio durante todo el período de muestreo.
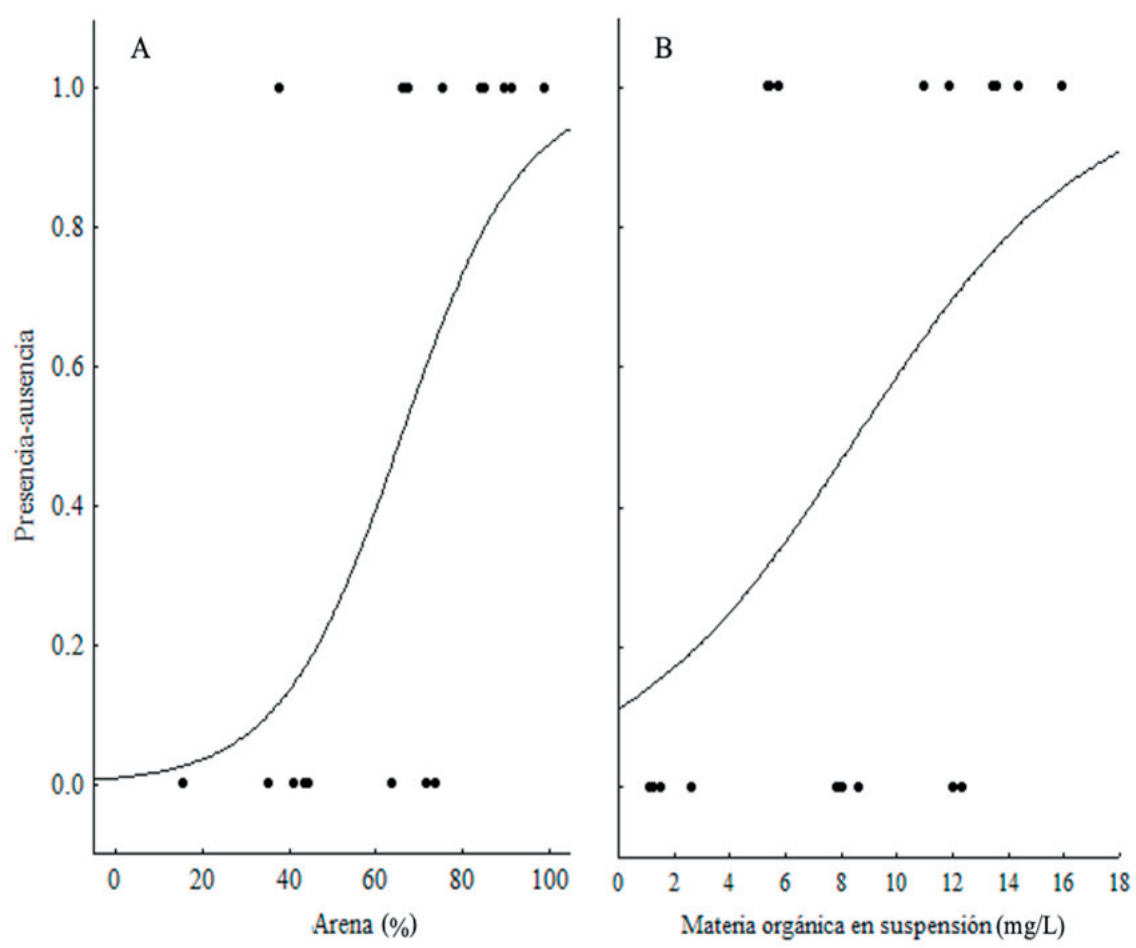

Figura 2. Curvas ajustadas para las regresiones logísticas de presencia-ausencia sobre porcentaje de arena en sedimentos (A) y materia orgánica en suspensión (B).

Figure 2. Adjusted curves for the presence-absence logistic regressions on percentage of sand (A) and organic matter in suspension (B). 


\section{Resultados}

\section{Muestreo piloto}

En un total de 18 sitios distribuidos en las cuencas del río Negro, Laguna Merín y río Santa Lucía (Figura 1), C. fluminea estuvo presente en el 50\% de los sitios (Apéndice 1). La presencia-ausencia de C. fluminea en todos los sitios mostró una relación significativa sólo con el porcentaje de arena $\left(\mathrm{Chi}^{2}=7.04\right.$, $P<0.01)$ y concentración de materia orgánica en suspensión $\left(\mathrm{Chi}^{2}=4.59, \mathrm{P}<0.05\right)$. Los valores de corte por encima de los cuales se espera que C. fluminea esté siempre presente fueron $65.9 \%$ de arena en el sedimento y $8.56 \mathrm{mg} / \mathrm{L}$ de materia orgánica en suspensión (Figura 2).

\section{Muestreos poblacionales}

Dos de las once variables ambientales consideradas (porcentaje de arena y porcentaje de grava), mostraron diferencias significativas entre sitios $(F=4.59, P<0.05)$. El porcentaje de arena en sedimentos aumentó con la distancia a la desembocadura, desde Santiago Vásquez hasta Florida (Figura 3). Santiago Vásquez fue el único sitio significativamente distinto de los demás (Figura 3).

El porcentaje de arena en sedimentos en San Ramón, el sitio situado más aguas arriba, fue significativamente menor que en Florida (Figura 3). Ambos sitios difieren significativamente de Santiago Vásquez, el sitio con el menor porcentaje de arena en sedimentos (LSD) (Figura 3). Los dos sitios restantes, Santa Lucía y Paso Pache, no presentaron diferencias significativas con ningún otro sitio (LSD) (Figura 3). Debido al bajo contenido de limo y arcilla en los sedimentos $(<2 \%)$, el comportamiento del porcentaje de grava en sedimentos fue inverso y complementario al porcentaje de arena, como es de esperarse cuando grava y arena son las fracciones dominantes en el sedimento. El porcentaje de grava disminuyó desde Santiago

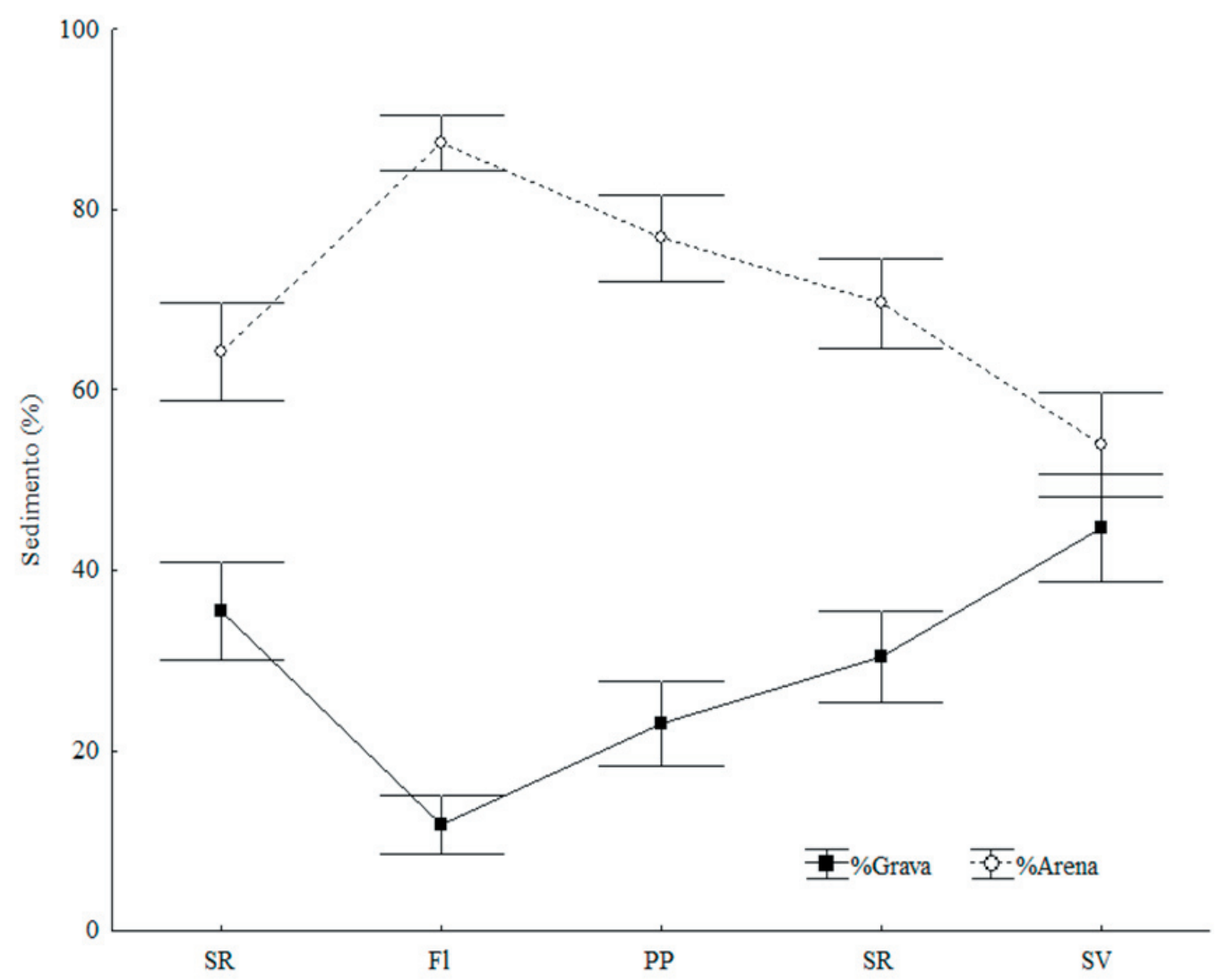

Figura 3. Variación en el porcentaje de grava y arena en sedimentos (mediaterror estandar) para los cinco sitios de muestreo en la cuenca del Santa Lucía, con datos promediados por sitio para todo el período de muestreo. Ver ubicación de localidades en Figura 1. Las letras iguales sobre y bajo los puntos indican la falta de diferencias significativas (prueba a posteriori $\mathrm{LSD}, P<0.05)$

Figure 3. Variation in gravel and sand percentages (mean \pm standard error) for the five sampling sites in the Santa Lucía basin, the data were averaged per site for the entire sampling period. See location of the sites in Figure 1. The letters above and below the points indicate the lack of significant differences (LSD a posteriori test, $P<0.05$ ) 
Vásquez hasta Florida y volvió a aumentar en San Ramón (Figura 3). Las diferencias entre sitios fueron significativas $(F=4.58, P<0.05)$ y las pruebas LSD indicaron los mismos grupos que en el caso anterior (Figura 3).

\section{Densidad}

En los cinco sitios de la cuenca del Santa Lucía se muestreó un total de 7161 individuos de C. fluminea. El mayor valor de densidad poblacional alcanzado fue de 1073 individuos/ $\mathrm{m}^{2}$ en febrero de 2002 para el sitio Florida (F), mientras que el menor valor fue de 6 individuos $/ \mathrm{m}^{2}$ en noviembre de 2001 para el sitio Santiago Vásquez (SV) (Figura 4). Los altos valores registrados en Florida y Paso Pache en febrero de 2002, en San Ramón durante julio de 2002 y en Santa Lucía en abril del mismo año (Figura 4), se debieron a la detección de alta densidad de individuos menores a 6 $\mathrm{mm}\left(>600\right.$ individuos $\left./ \mathrm{m}^{2}\right)$. En particular en
Florida, estos individuos aparecieron sólo en el subsitio banco, lo que explica el alto error estándar para ese sitio (Figura 4). En el sitio Santiago Vázquez no se registraron nunca individuos con longitud total menor a $8 \mathrm{~mm}$. En los cinco sitios, el banco fue en nueve de los once muestreos el subsitio con mayor densidad, mientras que el canal lo fue en agosto de 2001 y julio de 2002 y la orilla sólo lo fue en abril de 2002. La densidad (media \pm EE) difirió entre sitios $(F=11.29, P<0.001)$ (Tabla 1$)$, siendo significativamente menor en Santiago Vásquez $\left(15.7 \pm 4.6\right.$ individuos $\left./ \mathrm{m}^{2}\right)$ que en Florida $\left(301.4 \pm 100.4\right.$ individuos $\left./ \mathrm{m}^{2}\right)$, Paso Pache $\left(181.2 \pm 55.5\right.$ individuos $\left./ \mathrm{m}^{2}\right)$ y Santa Lucía (206.10 \pm 45.4 individuos $\left./ \mathrm{m}^{2}\right)$, mientras que en San Ramón $\left(158.6 \pm 38.5\right.$ individuos $\left./ \mathrm{m}^{2}\right)$ no difirió significativamente de ninguno de los anteriores grupos (LSD, $P<0.05$ ). No se encontraron diferencias significativas entre subsitios (Tabla 1), aunque se observó un comportamiento de aumento de la densidad

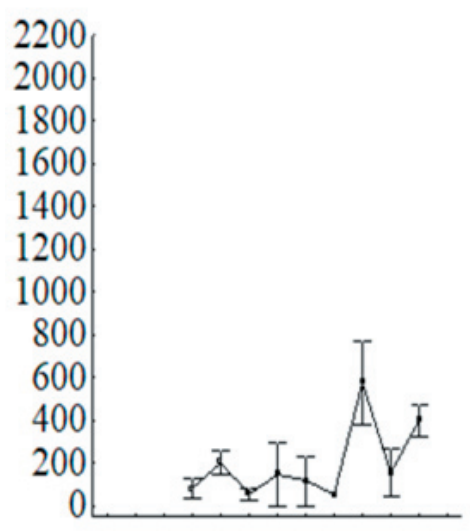

San Ramón

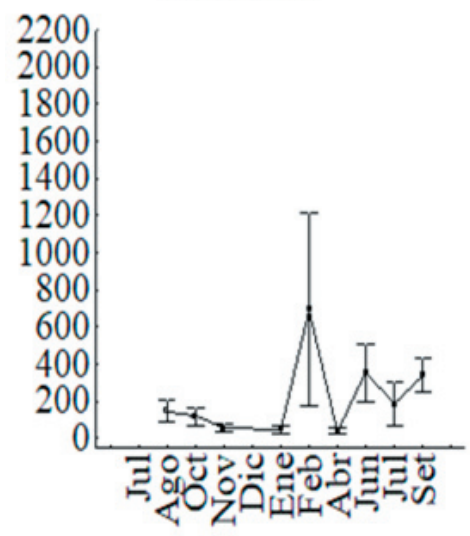

Paso Pache

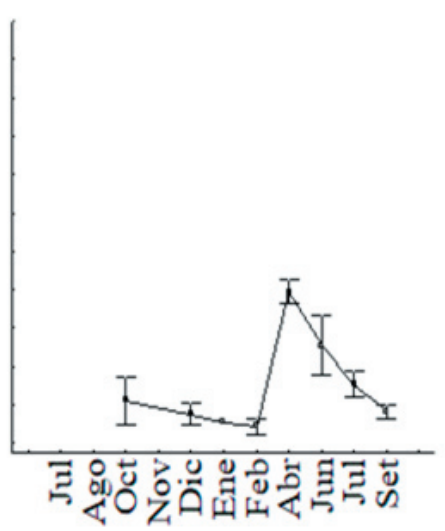

Santa Lucía

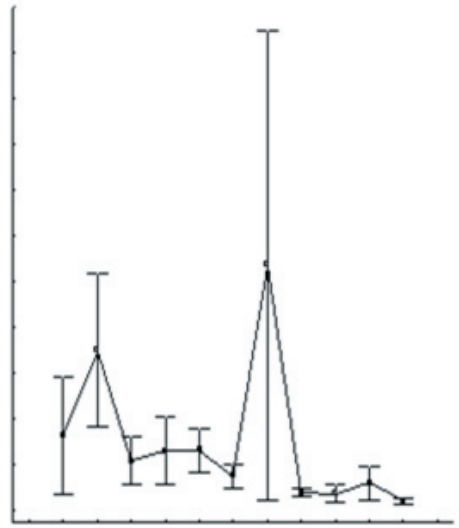

Florida

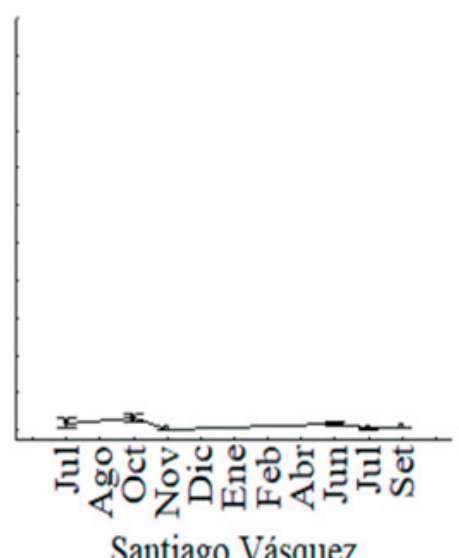

Santiago Vásquez

Figura 4. Variación temporal (mediaterror estandar) de la densidad de C. fluminea en los cinco sitios de la cuenca del Santa Lucía a lo largo del período de muestreo.

Figure 4. Temporal variation (mean \pm standard error) of C. fluminea density in the five sites in the Santa Lucía basin throughout the sampling period. 
Tabla 1. Resultados del ANOVA anidado (subsitio anidado en sitio) para los valores de densidad en los cinco sitios de la cuenca del río Santa Lucía a lo largo de todo el período de muestreo. SS: suma de cuadrados; gl: grados de libertad; MS: cuadrados medios. ${ }^{* *} P<0.01$; ${ }^{* * *} P<0.001$; ns: no significativo.

Table 1. Results of the nested ANOVA (subsite nested in site) for the density values in the five sites of the Santa Lucía river basin, throughout the entire sampling period. SS: sum of squares; gl: degrees of freedom; MS: average squares. ${ }^{* *} P<0.01 ;{ }^{* * *} P<0.001$; ns: not significant.

\begin{tabular}{lccccc}
\hline Efecto & SS & gl & MS & $F$ & \\
\hline Intercepto & 357.79 & 1 & 357.79 & 301.53 & $* * *$ \\
Subsitio & 4.80 & 2 & 2.40 & 2.02 & ns \\
Error & 35.60 & 30 & 1.19 & & \\
Sitio & 35.34 & 4 & 8.84 & 11.29 & $* * *$ \\
Sitio x Subsitio & 7.11 & 8 & 0.89 & 1.13 & ns \\
Error & 93.94 & 120 & 0.78 & & \\
\hline
\end{tabular}

Tabla 2. Parámetros de la ECVB obtenidos mediante ajuste no lineal para cada sitio de muestreo en la cuenca del río Santa Lucía. El parámetro de estacionalidad (C) se fijó en 1, dada la marcada estacionalidad de los datos analizados. ${ }^{*} P<0.05$; ${ }^{* *} P<0.01$; ${ }^{* * *} P<0.001 ;$ ns: no significativo; $L \infty$ : largo infinito; $\mathrm{K}$ : constante de crecimiento; $\mathrm{t}_{0}$ : tiempo subcero; WP: winter point.

Table 2. VBGE parameters obtained by non-linear adjustment for each sampling site in the Santa Lucía river basin. The seasonality parameter (C) was set at 1 , given the marked seasonality of the data analyzed. ${ }^{*} P<0.05 ;{ }^{*} P<0.01 ;{ }^{* *} P<0.001$; ns: not significant; $L \infty$ : infinite length; $\mathrm{K}$ : growth constant; $\mathrm{t}_{0}$ : subcero time; WP: winter point.

\begin{tabular}{lccccc}
\hline & $\begin{array}{c}\text { San } \\
\text { Ramón }\end{array}$ & Florida & $\begin{array}{c}\text { Paso } \\
\text { Pache }\end{array}$ & $\begin{array}{c}\text { Santa } \\
\text { Lucía }\end{array}$ & $\begin{array}{c}\text { Santiago } \\
\text { Vásquez }\end{array}$ \\
\hline $\mathrm{L} \infty(\mathrm{mm})$ & $20.14^{* * *}$ & $31.58^{* * *}$ & $17.34^{* * *}$ & $18.33^{* * *}$ & $34.83^{* * *}$ \\
$\left.\mathrm{~K}(\mathrm{año})^{-1}\right)$ & 2.65 & $0.60^{* *}$ & $3.18^{*}$ & $4.20^{* * *}$ & $1.03 \mathrm{~ns}$ \\
$\mathrm{t}_{0}(\mathrm{año})$ & $0.48^{* * *}$ & $0.04 \mathrm{~ns}$ & $0.40^{* * *}$ & $0.30^{* * *}$ & $-0.19 \mathrm{~ns}$ \\
$\mathrm{C}$ & 1.00 & 1.00 & 1.00 & 1.00 & 1.00 \\
$\mathrm{WP}$ & $0.90^{* * *}$ & $0.82^{* * *}$ & $0.82^{* * *}$ & $0.63^{* * *}$ & $0.81^{* * *}$ \\
$\mathrm{R}^{2}$ & $0.81^{* * *}$ & $0.94^{* * *}$ & $0.91^{* * *}$ & $0.96^{* * *}$ & $0.76^{* * *}$ \\
\hline
\end{tabular}

desde la orilla $\left(106.4 \pm 186.35\right.$ individuos $\left./ \mathrm{m}^{2}\right)$ hacia el banco $\left(285.6 \pm 499.76\right.$ individuos $\left./ \mathrm{m}^{2}\right)$.

\section{Crecimiento}

El análisis de frecuencias de tallas mediante el método de Bhattacharya identificó cuatro clases modales en todos los sitios (10-13 mm, 14-18 mm, 21-23 mm y 25-29 mm). La varianza explicada por el ajuste no lineal de la ECVB fue siempre mayor a 76\% (Tabla 2). Florida y Santiago Vásquez presentaron mayores valores de Lo(largo asintótico, el mayor tamaño alcanzado por la población) que los otros tres sitios, mientras que los valores de $\mathrm{K}$ (constante de crecimiento) $y t_{0}$ (tiempo subcero, el tiempo en el cual el individuo tendría tamaño cero) se comportaron de manera inversa (Tabla 2). Los valores de WP (winter point, el momento de menor crecimiento en el año) presentaron su mínimo en Santa Lucía y su máximo en San Ramón (Tabla 2).

La comparación mediante el LRT de las ECVB ajustadas para cada sitio mostró diferencias significativas para $L_{\infty}, K, t_{0} y W P$, entre Santiago Vásquez y los demás sitios (LRT: $P<0.001)$. Las combinaciones de parámetros que presentaron diferencias significativas fueron $\mathrm{L} \infty, \mathrm{K}$ y $\mathrm{t}_{0}$ y las combinaciones de cuatro parámetros (Tabla 3). Esto indica que las diferencias entre sitios se deberían fundamentalmente a las combinaciones de los parámetros de crecimiento más que a diferencias en los parámetros aislados de la ECVB.

\section{Mortalidad}

La tasa de mortalidad total $(Z)$ varió entre 4.51 año $^{-1}$ para San Ramón y 1.38 año $^{-1}$ para

Tabla 3. Valores de significancia $(P)$ para la comparación mediante LRT de los parámetros de las ECVB ajustadas para cada sitio. Sólo se presentan las combinaciones de parámetros que presentaron resultados significativos. Se utilizó un nivel de significancia, determinado por la relación de Bonferroni, de $\alpha=0.01$. CC: curvas coincidentes.

Table 3. Significance values $(P)$ for the comparison by LRT of the parameters of the VBGE adjusted for each site. Only the combinations of parameters that yield significant results are showed. Significance level, determined by the Bonferroni relation, was $\alpha=0.01$. CC: coincident curves.

\begin{tabular}{lcccc}
\hline & $\mathrm{CC}$ & $\mathrm{L}_{\alpha^{\prime}} \mathrm{K}, \mathrm{t}_{0}$ & $\mathrm{~L}_{\alpha^{\prime}} \mathrm{K}, \mathrm{t}_{0^{\prime}} \mathrm{C}$ & $\mathrm{L}_{\infty^{\prime}} \mathrm{K}, \mathrm{t}_{0^{\prime}}, \mathrm{WP}$ \\
\hline S. Ramón - Florida & 0.63 & 0.50 & 0.56 & 0.66 \\
S. Ramón - P. Pache & 0.96 & 0.99 & 1.00 & 1.00 \\
S. Ramón - S. Lucía & 0.56 & 0.10 & 0.14 & 0.19 \\
S. Ramón - S. Vásquez & $3.48 \mathrm{E}-04$ & $2.22 \mathrm{E}-04$ & $6.45 \mathrm{E}-04$ & $1.55 \mathrm{E}-04$ \\
Florida - P. Pache & 0.05 & 0.40 & 0.18 & 0.51 \\
Florida - S. Lucía & 0.02 & 0.22 & 0.71 & 0.36 \\
Florida - S. Vásquez & $3.54 \mathrm{E}-07$ & $9.29 \mathrm{E}-07$ & $3.34 \mathrm{E}-06$ & $8.12 \mathrm{E}-07$ \\
P. Pache - S. Lucía & 0.21 & 0.08 & 0.47 & 0.14 \\
P. Pache - S. Vásquez & $6.35 \mathrm{E}-05$ & $1.14 \mathrm{E}-04$ & $3.41 \mathrm{E}-04$ & $9.03 \mathrm{E}-05$ \\
S. Lucía - S. Vásquez & $2.01 \mathrm{E}-05$ & $7.18 \mathrm{E}-05$ & $2.20 \mathrm{E}-04$ & $1.46 \mathrm{E}-05$ \\
\hline
\end{tabular}


Tabla 4. Tasas de mortalidad total $\left(\mathrm{Z}\right.$ : año $\left.{ }^{-1}\right)$ y estadísticos asociados derivados de la curva de captura convertida a longitud ajustada para cada sitio de la cuenca del Santa Lucía. EE: error estándar. ${ }^{* * *} P<0.001$.

Table 4. Total mortality rates $\left(\mathrm{Z}\right.$ : year $\left.{ }^{-1}\right)$ and associated statistics derived from length converted catch curve adjusted for each site in the Santa Lucía basin. EE: standard error; ${ }^{* * *} P<0.001$.

\begin{tabular}{lcccccccccc}
\hline & S. Ramón & EE & Florida & EE & P. Pache & EE & S. Lucía & EE & S. Vásquez & EE \\
\hline Intercepto & 8.06 & 0.81 & 6.46 & 0.29 & 6.24 & 1.14 & 5.65 & 1.62 & 5.06 & 0.56 \\
$\mathrm{Z}\left(\mathrm{año}^{-1}\right)$ & 4.51 & 0.79 & 1.38 & 0.09 & 2.72 & 2.30 & 3.61 & 2.72 & 1.55 & 0.33 \\
$\mathrm{R}^{2}$ & $0.79^{* * *}$ & & $0.88^{* * *}$ & & $0.17^{* * *}$ & & $0.11^{* * *}$ & & $0.62^{* * *}$ & \\
\hline
\end{tabular}

Tabla 5. Resultados del análisis de regresión lineal múltiple de las variables ambientales sobre la densidad, mortalidad y parámetros de crecimiento. ${ }^{*} P<0.05$; ${ }^{* *} P<0.01$; ${ }^{* * *} P<0.001$; ns: no significativo; $\beta$ : coeficiente de correlación parcial.

Table 5. Results of multiple linear regression analysis of environmental variables on density, mortality and growth parameters. ${ }^{*} P<0.05 ;{ }^{* *} P<0.01 ;{ }^{* * *} P<0.001$; ns: not significant; $\beta$ : partial correlation coefficient.

\begin{tabular}{|c|c|c|c|c|c|c|}
\hline Variable dependiente & $\mathrm{R}^{2}$ ajustado & $F$ & & Variables incluidas & $\beta$ & \\
\hline \multirow[t]{3}{*}{ Densidad (individuos $/ \mathrm{m}^{2}$ ) } & 0.24 & 5.16 & ** & MOS (mg/L) & -0.32 & ns \\
\hline & & & & Temp $\left({ }^{\circ} \mathrm{C}\right)$ & -022 & ns \\
\hline & & & & Limo (\%) & -0.27 & ns \\
\hline \multirow[t]{2}{*}{$Z\left(a \tilde{n} o^{-1}\right)$} & 0.30 & 9.56 & $* * *$ & $\mathrm{Md}(\mathrm{mm})$ & 0.71 & $* * *$ \\
\hline & & & & Arena $(\%)$ & 0.24 & ns \\
\hline \multirow[t]{4}{*}{$\mathrm{L}_{\infty}(\mathrm{mm})$} & 0.25 & 4.17 & $* *$ & Grava (\%) & -0.55 & * \\
\hline & & & & Arena $(\%)$ & -0.31 & ns \\
\hline & & & & Limo (\%) & 0.33 & ns \\
\hline & & & & Stdev & -0.29 & ns \\
\hline \multirow[t]{3}{*}{$K\left(a \tilde{n} o^{-1}\right)$} & 0.15 & 3.25 & * & $\mathrm{Md}(\mathrm{mm})$ & 0.61 & $* *$ \\
\hline & & & & Arena $(\%)$ & -0.31 & ns \\
\hline & & & & Kur & -0.18 & ns \\
\hline \multirow[t]{4}{*}{$\mathrm{t}_{0}(\mathrm{año})$} & 0.34 & 6.11 & $* * *$ & Grava (\%) & 0.58 & * \\
\hline & & & & Arena $(\%)$ & 0.31 & ns \\
\hline & & & & Limo (\%) & -0.33 & ns \\
\hline & & & & Stdev & 0.39 & ns \\
\hline WP & 0.04 & 2.80 & ns & Kur & 0.26 & ns \\
\hline
\end{tabular}

Florida (Tabla 4). Si bien todas las regresiones fueron significativas $(P<0.001)$, la varianza explicada para Paso Pache y Santa Lucía fue notablemente menor que para los otros tres sitios (Tabla 4).

\section{Relación entre variables ambientales y poblacionales}

Las regresiones múltiples fueron significativas, exceptuando las que tuvieron a la densidad y a WP como variables dependientes (Tabla 5). Para las variables dependientes restantes, aunque siempre fue introducida más de una variable, en cada caso solo una resultó significativa (Tabla 5). Por lo tanto, los mejores predictores para la densidad, $\mathrm{Z}, \mathrm{L}_{\infty}, \mathrm{K}$ y $\mathrm{t}_{0}$ fueron el diámetro medio de grano $(\beta=0.71 ; P<0.001)$, el porcentaje de grava en sedimentos $(\beta=-0.55 ; P<0.05)$, el diámetro medio de grano $(\beta=0.61 ; P<0.01)$ y el porcentaje de grava en sedimentos $(\beta=0.58$; $P<0.05$ ), respectivamente (Tabla 5). Las regresiones lineales de $\mathrm{L}_{\alpha^{\prime}} \mathrm{K}, \mathrm{t}_{0^{\prime}}$ WP y $\mathrm{Z}$ sobre la densidad no fueron significativas, lo cual indica que los valores de estos parámetros no varían junto con la densidad poblacional.

\section{Discusión}

\section{Distribución de C. fluminea en Uruguay}

La incidencia de la almeja asiática en la región Centro-Sur y Este de Uruguay fue de $50 \%$. La mitad de los sitios relevados fueron colonizados por C. fluminea, lo cual demuestra la gravedad de la invasión. El patrón de presencia-ausencia encontrado en las cuencas relevadas concuerda con la forma de arribo de la especie a la región, propuesta en la literatura (Amestoy et al. 1998; Ituarte 1981). El primer reporte fue en la localidad de Punta Lara, en la costa argentina del Río de la Plata (Ituarte 1981). Desde allí, C. fluminea extendió su distribución hacia el norte hasta el río Paraná y al sur hasta la ciudad de Magdalena. Los 
primeros registros en Uruguay fueron en los departamentos de Colonia y San José (Veintenheimer-Mendes and Olazarri 1983). Luego, se encontraron ejemplares en el río Uruguay y en el río Negro (Amestoy et al. 1998). La dispersión contracorriente de $C$. fluminea se puede explicar por la mediación de peces que depredan sobre la almeja, pudiendo de este modo desplazarse hasta $1.2 \mathrm{~km}$ aguas arriba por año sin intervención humana (Voelz et al. 1998).

De esta forma, C. fluminea habría avanzado aguas arriba del río Negro, superando las represas de Palmar, Baygorria y Rincón del Bonete, seguramente mediante facilitación humana debido a su uso como carnada (Counts 1986; Argente 2016), hasta llegar a la localidad del kilómetro 329. La invasión también habría proseguido su avance por la costa del Río de la Plata, llegando hasta la desembocadura del río Santa Lucía, el que, a su vez, habría sido invadido. La dispersión a favor de la corriente se ve facilitada por la producción de una línea de arrastre mucosa por células de las demibranquias internas de juveniles y adultos jóvenes. Se ha probado que esta línea de arrastre contribuye a la flotabilidad de las almejas de hasta $14 \mathrm{~mm}$ de longitud total, promoviendo la deriva hacia nuevas localidades y favoreciendo la dispersión de la especie (Rosa et al. 2014). Sin embargo, debido a que C. fluminea no soporta salinidades mayores de 14-15 (Morton and Tong 1985; Argente 2016; Bertrand et al. 2017), las cuales son comunes frente al departamento de Montevideo (Nagy et al. 1998), la invasión no podría avanzar más allá de sus costas. Otra especie invasora de la región, el mejillón dorado (Limnoperna fortunei), también presenta su límite este de distribución en esta zona (Brugnoli et al. 2005). El gradiente de salinidad generado por la interacción del Río de la Plata y el océano Atlántico constituiría el principal factor limitante de la distribución espacial de ambas especies. En base a la tolerancia a la salinidad anteriormente descripta, la invasión de la cuenca de la laguna Merín por C. fluminea tendría un origen diferente, probablemente a través del canal de San Gonzalo, desde la zona estuarina de la laguna de los Patos, del mismo modo que se ha planteado para la invasión de la laguna Merín por L. fortunei (Langone 2005b). Las condiciones estuarinas reinantes en la zona del canal de San Gonzalo habrían retrasado el pasaje de L. fortunei, que no soporta salinidades superiores a 3 (Darrigran 2002) hacia la laguna Merín (Brugnoli et al.
2005), pero no constituirían un obstáculo para el pasaje de $C$. fluminea.

La probabilidad de ocurrencia y densidad poblacional de $C$. fluminea estuvieron determinadas en gran medida por el porcentaje de arena en el sustrato, con densidades decrecientes tanto hacia sedimentos más finos (limos-arcillas) como hacia más gruesos (gravas). Esto confirma la preferencia de $C$. fluminea por sedimentos arenosos (ver Britton and Morton 1982; Karatayev et al. 2003; Clavijo 2014; Patrick et al. 2017). La densidad poblacional fue significativamente menor en Santiago Vásquez que en los otros cuatro sitios. Esto puede atribuirse a la granulometría, ya que Santiago Vásquez presentó en sus sedimentos un mayor porcentaje de fracciones finas que los otros cuatro sitios. Dicho sedimento fino es más compacto, lo que dificulta la excavación e, incluso, la apertura y cierre de las valvas de estas almejas (Newell and Hidu 1982). Además, los porcentajes de limo hallados en Santiago Vásquez podrían reducir las tasas de filtración por colmatación de las branquias, dificultar el crecimiento al introducirse entre el manto y el caparazón o incluso sepultar y ahogar por completo a algunos individuos (Appeldoorn 1983; Tuttle-Raycraft 2017). Un alto porcentaje de grava también es contraproducente, ya que los granos de sedimento más grandes pueden dañar las valvas o bien dificultar el movimiento a través del sedimento (Appeldoorn 1983), lo cual podría causar un aumento en la mortalidad.

\section{Crecimiento}

Los valores de los parámetros de la ECVB sugieren que Santiago Vásquez y Florida se separan de Paso Pache, San Ramón y Santa Lucía. Sin embargo, la comparación de las curvas de crecimiento mediante la prueba de LRT estimó diferencias significativas entre Santiago Vásquez y los demás sitios. Esto podría ser explicado por diferencias ambientales, en particular aquellas referidas a la granulometría (\% de arena) y concentración de materia orgánica en suspensión. A su vez, la relación positiva entre la concentración de materia orgánica en suspensión y el parámetro $\mathrm{L}_{\infty}$ de la ECVB permite esperar mayores valores de $\mathrm{L}_{\infty}$ aguas abajo, donde la concentración de alimento es mayor (Vannotte et al. 1980). El parámetro de curvatura $\mathrm{K}$ se relacionó de forma positiva con el diámetro medio de grano, por lo cual cabe esperar menores valores de $\mathrm{K}$ aguas abajo, donde 
dicho diámetro es menor (Elliott 2010). Esta combinación de parámetros permite esperar aguas abajo individuos con mayor $\mathrm{L}_{\infty} \mathrm{y}$ menor $\mathrm{K}$, cuyo crecimiento es más lento y alcanzan mayor tamaño, mientras que los individuos de los sitios aguas arriba tenderían a un crecimiento más rápido y un menor tamaño.

Los tres sitios que presentaron menores $\mathrm{L}_{\infty} \mathrm{y}$ mayores valores de K (i.e., San Ramón, Paso Pache y Santa Lucía) son también aquellos que durante los meses de verano sufrieron bajantes importantes, lo que afecta el valor y la variabilidad de la tasa de mortalidad (Byrne et al. 1990). Esto podría llevar a que los individuos de dichos sitios tengan una menor esperanza de vida $\mathrm{y}$, por lo tanto, alcancen menores longitudes. También son aquellos sitios en los cuales durante los muestreos se constató que se extrae arena para la construcción, con el consiguiente efecto sobre las poblaciones de C. fluminea. La mayor frecuencia de los disturbios en estos sitios ocasionaría que estas poblaciones se acerquen más a una estrategia $\mathrm{r}$, presentando un menor largo asintótico y una mayor constante de crecimiento que las poblaciones de los sitios más estables. Según Britton y Morton (1982), C. fluminea es capaz de adoptar estrategias $\mathrm{K}$ o r según habite en ambientes estables o frecuentemente perturbados.

Según McMahon y Williams (1986), la temperatura fue el factor ambiental más importante que afectó el crecimiento de C. fluminea. Cabe señalar que, aún cuando las diferencias en temperatura no fueron significativas entre sitios debido a que el rango geográfico considerado en este estudio fue muy reducido, esta variable explicó el $50 \%$ de la variación en el parámetro WP de la ECVB. Esto sugiere que la temperatura determinaría fluctuaciones intra-anuales en el crecimiento.

\section{Mortalidad}

Gran parte de la variación en el coeficiente instantáneo de mortalidad total (Z) fue explicada por la granulometría y en particular el diámetro medio de grano: a mayor diámetro medio de grano mayor mortalidad. Las propiedades abrasivas del sedimento aumentan junto con el diámetro medio de grano, lo cual podría causar un mayor desgaste de las valvas y una mayor mortalidad.

Además, la mortalidad se vio influenciada, en menor grado, por la concentración de materia orgánica en suspensión, la temperatura y la concentración de $\mathrm{O}_{2}$. El óptimo de temperatura de $C$. fluminea se ubica alrededor de $25^{\circ} \mathrm{C}$, y se consideran letales los valores superiores a $34-32{ }^{\circ} \mathrm{C}$ e inferiores a $2{ }^{\circ} \mathrm{C}$ (Janech and Hunter 1985; Mattice and Wright 1986; Argente 2016). Las temperaturas elevadas se relacionan con un menor contenido de $\mathrm{O}_{2}$ en el agua, y C. fluminea es intolerante a los bajos niveles de oxígeno (Buttner 1986; McMahon 2000; Argente 2016). Por otra parte, las bajas temperaturas inhiben la formación del biso en los juveniles, o incluso disminuyen su capacidad de excavación (Williams and McMahon 1986), lo cual aumenta la posibilidad de que sean arrastrados por la corriente. Las temperaturas entre 7 y $20{ }^{\circ} \mathrm{C}$ registradas durante este estudio se acercaron más al límite inferior del rango térmico de $C$. fluminea que al superior. Los menores valores de temperatura se registraron en San Ramón, en coincidencia con los mayores valores de $Z$, lo que podría indicar que las bajas temperaturas causan un aumento en la mortalidad.

\section{Conclusiones}

La presencia de C. fluminea estuvo correlacionada con el porcentaje de arena en el sedimento y la concentración de materia orgánica en suspensión. Se espera que $C$. fluminea no esté presente si el porcentaje de arena en sedimentos es menor a $66 \%$ o la concentración de materia orgánica en suspensión cae por debajo de $8.6 \mathrm{mg} / \mathrm{L}$.

La densidad poblacional estuvo explicada en mayor grado por factores granulométricos, aumentando con el porcentaje de arena y disminuyendo con el de grava. La expansión de C. fluminea estaría limitada a aquellos sectores de los cursos de agua donde el porcentaje de arena en sedimentos es dominante y tanto la grava como el limo mantienen bajos sus porcentajes.

Tanto la variación en los parámetros de la ECVB como en la tasa de mortalidad total Z fueron explicados en su mayoría por variables granulométricas. Los sedimentos finos promueven el crecimiento lento e individuos de gran tamaño y una menor mortalidad, mientras que los sedimentos gruesos llevan a crecimiento más rápido con individuos más pequeños y altas mortalidades.

Se espera que la especie siga avanzando en los cursos de agua en los que ya se encuentra e invada todos los que se conectan con ellos, 
hasta encontrar condiciones granulométricas desfavorables. En particular, se predice que el río Yí y sus afluentes, en los cuales no se registró presencia de $C$. fluminea, serán ocupados al menos hasta la zona de Durazno (ver Figura 1), en la cual existen arenales extensos. De igual modo, se espera que la distribución continúe extendiéndose en la cuenca de la Laguna Merín, ocupando los ríos Tacuarí y Olimar (ver Figura 1).

\section{REFERENCIAS}

Agudo-Padrón, A. I. 2017. Another invader among us: first confirmed record of the invasive non-native Asian clam Corbicula fluminalis (Müller, 1774) in Santa Catarina state, Southern Brazil. Ellipsaria 19:28-30.

Amestoy, F., M. Spinetti, and G. Fabiano. 1998. Aquatic species introduced in Uruguay. Verhandlungen aus die International Vereinigung für Theoretisch und Anwenden Limnologie 26:2170-2173. https://doi.org/10.1080/ 03680770.1995.11901129.

Appeldoorn, R. S. 1983. Variation in the growth rate of Mya arenaria and its relationship to the environment as analyzed trough principal components analysis and the $\omega$ parameter of the von Bertalanffy equation. Fishery Bulletin 8:7584.

Argente, F. A. T. 2016. Bivalve Superpower: The global invasion of Corbiculid clams. Annual Research and Review in Biology 10:1-10. https://doi.org/10.9734/ARRB/2016/26448.

Arocena, R., and D. Conde. 1999. Métodos en ecología de aguas continentales con ejemplos de limnología en Uruguay. DIRAC Facultad de Ciencias, Montevideo, Montevideo, Uruguay.

Barone, L. R., C. E. Rodríguez, M. L. Ghiglioni, C. D. Gonzáles, and S. S. Luna. 1994. Uruguay, mi país: geografía y vida. Cultural Librera Americana S.A., Buenos Aires, Buenos Aires, Argentina.

Begon, M., C. Townsend, and J. Harper. 1996. Ecology: individuals, populations and communities. Blackwell Science Ltd., Oxford, Oxforshire, UK.

Bertrand, C., S. Devina, C. Mouneyrac, and L. Giambérini. 2017. Eco-physiological responses to salinity changes across the freshwater-marine continuum on two euryhaline bivalves: Corbicula fluminea and Scrobicularia plana. Ecological Indicators 74:334-342. https://doi.org/10.1016/j.ecolind.2016.11.029.

Bresciano, D., C. Rodríguez, F. Lezama, and A. Altesor. 2014. Patrones de invasión de los pastizales de Uruguay a escala regional. Ecología Austral 24:83-93. https://doi.org/10.25260/EA.14.24.1.0.40.

Britton, J. C., and B. Morton. 1982. A dissection guide, field and laboratory manual for the introduced bivalve Corbicula fluminea. Malacological Review 3:1-82.

Brugnoli, E., J. Clemente, L. Boccardi, A. Borthagaray, and F. Scarabino. 2005. Golden mussel Limnoperna fortunei (Bivalvia: Mytilidae) distribution in the main hydrographical basins of Uruguay: update and predictions. Anais da Academia Brasileira de Ciências 77:235-244. https://doi.org/10.1590/S0001-37652005000200004.

Buttner, J. K. 1986. Biology of Corbicula in catfish rearing ponds. American Malacological Bulletin Special Edition 2: 211-218.

Byrne, R. A., E. Gnaiger, R. F. McMahon, and T. H. Dietz. 1990. Behavioral and metabolic responses to emersion and subsequent reimmersion in the freshwater bivalve, Corbicula fluminea. Biological Bulletin 178:251-259. https: //doi.org/10.2307/1541826.

Cao, L., C. Damborenea, P. E. Penchaszadeh, and G. Darrigran. 2017. Gonadal cycle of Corbicula fluminea (Bivalvia: Corbiculidae) in Pampean streams (Southern Neotropical Region). PLoS ONE 12:1-16. https://doi.org/10.1371/ journal.pone.0186850.

Chapin, F. S., E. S Zavaleta, V. T. Eviner, R. L. Naylor, P. M. Vitousek, H. L. Reynolds, D. U. Hooper, S. Lavorel, O. E. Sala, S. E. Hobble, M. C. Mack, and S. Díaz. 2000. Consequences of changing biodiversity. Nature 405:234-242. https: //doi.org/10.1038/35012241.

Cherry, D. S., J. Cairns, and R. L. Graney. 1980. Asiatic clam invasion. Causes and effects. Water Spectrum 12:19-24.

Clavijo, C. 2014. Diversidad de Corbiculidae (Mollusca: Bivalvia) en Uruguay. Tesis de Maestría. PEDECIBA Facultad de Ciencias UdelaR, Montevideo. Uruguay. Pp. 122.

Clemente, J., and E. Brugnoli. 2002. First record of Limnoperna fortunei (Dunker 1857) (Bivalvia: Mytilidae) in continental waters of Uruguay. Boletín de la Sociedad Zoológica Uruguay 13:29-33.

Counts, C. L. 1986. The zoogeography and history of the invasion of the United States by Corbicula fluminea (Bivalvia: Corbiculidae). American Malacological Bulletin 2:7-39.

Crooks, J. A. 2002. Characterizing ecosystem-level consequences of biological invasions: the role of ecosystem engineers. Oikos 97:153-166. https://doi.org/10.1034/j.1600-0706.2002.970201.x.

Daroczi, I., E. García, and M. Liguera. 1983. Atlas para la República Oriental del Uruguay. Ediciones Raschetti Ltda./ Ediciones de Montevideo SRL, Primera edición. Montevideo, Montevideo, Uruguay.

Darrigran, G., and C. Damborenea. 2011. Ecosystem engineering impacts of Limnoperna fortunei in South America. Zoological Science 28:1-7. https://doi.org/10.2108/zsj.28.1.

Darrigran, G. A., and M. E. Maroñas. 1989. Crecimiento de las poblaciones naturales de Corbicula fluminea (Muller, 1774) y C. largillierti (Phillippi, 1844) (Bivalvia: Sphaeriacea) en el litoral de Punta Blanca, estuario del Río de la Plata, República Argentina. Comunicaciones de la Sociedad Malacológica del Uruguay 56-57:139-157.

Darrigran, G. 1992a. Nuevos datos acerca de la distribución de las especies del género Corbicula (Bivalvia, Sphaeriacea) en el área del Río de la Plata, República Argentina. Notas Museo La Plata 21:143-148. 
Darrigran, G. 1992b. Variación temporal y espacial de la distribución de las especies del género Corbicula Megerle, 1811 (Bivalvia, Corbiculidae) en el estuario del Río de la Plata, República Argentina. Neotropica 38:59 63.

Darrigran, G. 2002. Potential impact of filter-feeding invaders on temperate inland freshwater environments. Biological Invasion 1-2:145-156. https://doi.org/10.1023/A:1020521811416.

Davis, M. A., and K. Thompson. 2000. Eight ways to be a colonizer; two ways to be an invader: a proposed nomenclature scheme for invasion ecology. Bulletin of the Ecological Society of America 81:226-230.

Davis, M. A., J. P. Grime, and K. Thompson. 2000. Fluctuating resources in plant communities: a general theory of invasibility. Journal of Ecology 88:528-534. https://doi.org/10.1046/j.1365-2745.2000.00473.x.

Elliott, S. 2010. El río y la forma: Introducción a la geomorfología fluvial. Ril Editors, Santiago, Santiago, Chile.

Folk, R. L., and W. C. Ward. 1957. Brazos River Bar: A study in the significance of grain size parameters. Journal of Sedimentology and Petrology 3:26-27. https://doi.org/10.1306/74D70646-2B21-11D7-8648000102C1865D.

Foster, A. M., P. Fuller, A. Benson, S. Constant, D. Raikow, J. Larson, and A, Fusaro. 2012. Corbicula fluminea. USGS Non-indigenous Aquatic Species Database, Gainesville. URL: nas.er.usgs.gov/queries/FactSheetList.aspx.

Gayanilo, F. C., P. Sparre, and D. Pauly. 2002. The FISAT II user's guide. FAO, Roma, Lacio, Italy.

Haddon, M. 2001. Modeling and quantitative methods in fisheries. Chapman and Hall/CRC, Boca Raton, Florida, USA.

Hoffmann, B. D., and F. Courchamp. 2016. Biological invasions and natural colonisations: are they that different? NeoBiota 29:1-14. https://doi.org/10.3897/neobiota.29.6959.

Isom, B. G. 1986. Historical review of Asiatic clam (Corbicula) invasion and biofouling of waters and industries in the Americas. American Malacological Bulletin Special Edition 2:1-15.

Ituarte, C. F. 1981. Primera noticia acerca de la introducción de pelecípodos asiáticos en el área rioplatense. Neotrópica 27:79-82.

Ituarte, C. F. 1982. Contribución al conocimiento de la biología de la familia Corbiculidae (Mollusca, Pelecypoda) en el Río de la Plata. Tesis Doctoral. Facultad de Ciencias Naturales y Museo Universidad Nacional de La Plata, La Plata, Buenos Aires, Argentina. Pp. 129.

Ituarte, C. F. 1994. Corbicula and Neocorbicula (Bivalvia: Corbiculidae) in the Paraná, Uruguay and Río de la Plata basins. The Nautilus 107:129-135.

Janech, M. G., and R. D. Hunter. 1985. Corbicula fluminea in a Michigan River: Implications for low temperature tolerance. Malacological Review 28:199-124.

Karatayev, A. Y., L. Burlokova, T. Kesterson, and D. Padilla. 2003. Dominance of the asiatic clam, Corbicula fluminea (Muller), in the benthic community of a reservoir. Journal of Shellfish Research 22:487-493.

Kimura, D. K. 1980. Likelihood methods for the von Bertalanffy growth curve. Fishery Bulletin 77:765-775.

Kurucz, A., A. Masello, S. Méndez, R. Cranston, and P. G. Wells. 1998. Calidad ambiental del Río de la Plata. En P. G. Wells and G. R. Daborn (eds.). El Río de la Plata: una revisión ambiental. Un informe de antecedentes del Proyecto EcoPlata. Dalhousie University, Halifax, Nova Scotia, Canada.

Langone, J. A. 2005a. Notas sobre el mejillón dorado Limnoperna fortunei (Dunker, 1857) (Bivalvia, Mytilidae) en Uruguay. Publicación Especial de la Sociedad Zoológica del Uruguay (Actas de las VIII Jornadas de Zoología del Uruguay), Montevideo, Montevideo, Uruguay.

Langone, J. A. 2005b. Notas sobre el mejillón dorado Limnoperna fortunei (Dunker 1857) (Bivalvia, Mytilidae) en Uruguay. Publicación Extra en línea. Museo Nacional de Historia Natural y Antropología 1:1-18.

Mansur, M., and L. Garces. 1988. Ocorrência e densidade de Corbicula fluminea (Múller, 1774) e Neocorbicula limosa (Maton, 1811) na Estação Ecológica do Taim e áreas adjacentes, Rio Grande do Sul, Brasil (Mollusca, Bivalvia, Corbiculidae). Iheringia, Serie Zoológica 68:99-115.

Mattice, J. S., and L. L. Wright. 1986. Aspects of growth of Corbicula fluminea. American Malacological Bulletin Special Edition 2:167-178.

Mazia, C. N., E. J. Chaneton, C. M. Ghersa, and R. J. C. León. 2001. Limits to tree species invasion in pampean grassland and forest plant communities. Oecologia 128:594-602. https://doi.org/10.1007/s004420100709.

McDonald, B. A., and R. J. Thompson. 1985. Influence of temperature and food availability on the ecological energetics of the giant scallop Placopecten magellanicus. 1: Growth rate of shell and somatic tissue. Marine Ecology Progress Series 25:279-294. https://doi.org/10.3354/meps025279.

McMahon, R. F., and C. J. Williams. 1986. A reassessment of growth rate, life span, life cycles and population dynamics in a natural population of Corbicula fluminea (Müller) (Bivalvia, Corbiculacea). American Malacological Bulletin Special Edition 2:151-166.

McMahon, R. F. 2000. Invasive characteristics of the freshwater bivalve Corbicula fluminea. Pp. 315-343 in R. Claudi and J. Leach (eds.). Non-Indigenous Freshwater Organisms: Vectors, Biology and Impacts. First edition. Lewis Publishers, Boca Raton, Florida, USA.

Morales, J., F. Flechoso, M. Lizana, and A. Negro. 2013. Patrones de colonización y ecología de poblaciones de dos bivalvos invasores (mejillón cebra Dreissena polymorpha Pallas, 1771 y almeja asiática Corbicula fluminea Müller, 1774) en un tramo lótico del Ebro medio (Castejón, Navarra). Munibe (Ciencias Naturales-Natur Zientziak) 61:47-69.

Morton, B. 1973. Some aspects of the biology and functional morphology of the organs of feeding and digestion of Limnoperna fortunei (Dunker) (Bivalvia: Mytilacea). Malacologia 12:265-281.

Morton, B. 1977. Freshwater fouling bivalves. Proceedings of the International Corbicula Simposium. Texas Christian 
University Research Foundation, Fort Worth, Texas, USA.

Morton, B. 1986. Corbicula in Asia. An updated Synthesis. American Malacological Bulletin Special Edition 2:113-124.

Morton, B., and K. Y. Tong. 1985. The salinity tolerance of Corbicula fluminea (Bivalvia: Corbiculoidea) from Hong Kong. Malacological Review 18:91-95.

Nagy, G. J., C. M. Martínez, R. M. Caffera, G. Pedrosa, E. A. Forbes, A. C. Perdomo, and J. P. Laborde. 1998. Marco hidrológico y climático del Río de la Plata. En P. G. Wells and G. R. Daborn (eds.). El río de La Plata: una revisión ambiental. Un informe de antecedentes del Proyecto EcoPlata. Dalhousie University, Halifax, Nova Scotia, Canada.

Newell, C. R., and H. Hidu. 1982. The effects of sediment type on growth rate and shell allometry in the soft-shelled clam Mya arenaria. Journal of Experimental Biology and Ecology 65:285-295. https://doi.org/10.1016/0022-0981(82)900600 .

Olazarri, J. 1986. Almejas del género Corbicula en el río Uruguay y sus afluentes del margen izquierdo. Pp. 65-67 en Seminario "El río Uruguay y sus recursos pesqueros". Primera edición. Comisión Administradora del Rio Uruguay (CARU), Paysandú, Paysandú, Uruguay.

Palacios, R., D. A. Armstrong, and J. M. Orensanz. 2000. Fate and legacy of an invasion: extinct and extant populations of the soft-shell clam in Grays Harbor (Washington). Aquatic Conservation: Marine and Freshwater Ecosystems 10: 279-303. https://doi.org/10.1002/1099-0755(200007/08)10:4\%3C279::AID-AQC412\%3E3.0.CO;2-I.

Parodiz, J. J., and L. Hennings. 1963. The Neocorbicula (Mollusca, Pelecypoda) of the Paraná-Uruguay basin, South America. Annals of Carnegie Museum 38:69-95.

Patrick, C. H., M. N. Waters, and S. W. Golladay. 2017. The distribution and ecological role of Corbicula fluminea (Müller, 1774) in a large and shallow reservoir. Bio Invasions Records 6:39-48. https://doi.org/10.3391/bir.2017.6.1.07.

Pauly, D., and J. F. Caddy. 1985. A modification of Bhattacharya's method for the analysis of mixtures of normal distributions. Fisheries Circulars № 781, FAO, Roma, Lacio, Italy.

Pauly, D., and G. Gaschütz. 1979. A simple method for fitting oscilating length grow data, with a program for pocket calculators. Interviews of the Council for Exploration of the Sea Committee Meetings (Demersal Fish Committee) 24:1-26.

Reshaid, Y., L. Cao, F. Brea, M. Ortiz Blanche, S. Torres, and G. Darrigran. 2017. Variation in distribution of Corbicula species (Mollusca, Bivalvia, Corbiculide) after 25 years in the Río de la Plata, Argentina. Zoologia 34:1-6. https: //doi.org/10.3897/zoologia.34.e22181.

Rodríguez, M., and R. Palacios. 2001. Presencia y abundancia de la almeja invasora Corbicula fluminea (Bivalvia: Corbiculidae) en ríos del Uruguay. Publicación Especial de la Sociedad Zoológica del Uruguay, Actas de las VI Jornadas de Zoología del Uruguay 1:60-61.

Rosa, I. C., J. L. Pereira, R. Costa, J. Gomes, M. L. Pereira, and F. Gonçalves. 2014. Dispersal of Corbicula fluminea: factors influencing the invasive clam's drifting behavior. Annales de Limnologie-International Journal of Limnology 50:37-47. https://doi.org/10.1051/limn/2013069.

Sakai, A. K., F. W. Allendorf, J. S. Holt, D. M. Lodge, J., Molofsky, K. A. With, S. Baughman, R. J. Cabin, J. E. Cohen, N. C. Ellstrand, D. E. McCauley, P. O'Neil, I. M. Parker, J. N. Thompson, and S. G. Weller. 2001. The population ecology of invasive species. Annual Reviews of Ecology and Systematic 32:305-332. https://doi.org/10.1146/annure v.ecolsys.32.081501.114037.

Shigesada, N., and K. Kawasaki. 1997. Biological invasions: theory and practice. Oxford University Press, Oxford, Oxfordshire, UK.

Simberloff, D., L. Souza, M. A. Núñez, M. N. Barrios-García, and W. Bunn. 2012. The natives are restless, but not often and mostly when disturbed. Ecology 93:598-607. https://doi.org/10.1890/11-1232.1.

Somers, I. F. 1988. On a seasonally oscillating growth function. Fishbyte 6:8-11.

Sousa, R., J. L. Gutiérrez, and D. C. Aldridge. 2009. Non-indigenous invasive bivalves as ecosystem engineers. Biological Invasions 11:2367-2385. https://doi.org/10.1007/s10530-009-9422-7.

Strauss, S. Y., C. O. Webb, and N. Salamin. 2006. Exotic taxa less related to native species are more invasive. Proceedings of the National Academy of Sciences 103:5841-5845. https://doi.org/10.1073/pnas.0508073103.

Sugio, K. 1973. Introduçao à sedimentologia. Edgar Blücher/EDUSP, São Paulo, Brasil.

Tuttle-Raycraft, S., T. J. Morris, and J. D. Ackerman. 2017. Suspended solid concentration reduces feeding in freshwater mussels. Science of the Total Environment 598:1160-1168. https://doi.org/10.1016/j.scitotenv.2017.04.127.

Vanotte, R. L., G. W. Minshall, K. W. Cummins, J. R. Sedell, and C. E. Gushing. 1980. The river continuum concept. Canadian Journal of Fisheries and Aquatic Sciences 37:130-137. https://doi.org/10.1139/f80-017.

Veintenheimer-Mendes, I., and J. Olazarri. 1983. Registros de Corbicula Megerle, 1811 (Bivalvia, Corbiculidae), para el río Uruguay. Boletín de la Sociedad Zoológica del Uruguay 1:50-53.

Voelz, N. J., J. V. McArthur, and R. B. Rader. 1998. Upstream mobility of the Asiatic clam Corbicula fluminea: Identifying potential dispersal agents. Journal of Freshwater Ecology 13:39-45. https://doi.org/10.1080/02705060.1998.9663589.

Williams, C. J., and R. F. McMahon. 1986. Power station entrainment of Corbicula fluminea (Müller) in relation to population dynamics, reproductive cycle and biotic and abiotic variables. American Malacological Bulletin Special Edition 2:99-111.

Zar, J. H. 1996. Biostatistical analysis. Third edition. Prentice-Hall Inc, Upper Saddle River, New Jersey, USA. 